\title{
JAVA WEB DEPLOYMENT IN CLOUD COMPUTING
}

\author{
Ankit Kumar Sahu \\ Department of Computer Science Engineering \\ Gyan Vihar School of Engineering and Technology, \\ Jaipur, Rajasthan, India.
}

\begin{abstract}
Cloud Computing is a revolutionary IT field in today's world. Several technologies like java, python provides deployment on clouds using respective tools. In this paper, the web deployment in cloud computing with java and the performance issues of the java deployment on a cloud is discussed.

There are several tools available to deploy a java webapplication on clouds, some of these are Google App Engine (By Google), Windows Azure (By Microsoft), Amazon EC2 (By Amazon) etc. Cloud Computing is providing many facilities for deployment but is also having performance issues which is a major factor for the web-applications. Issues with java-deployment on cloud would try to resolve through the framework customization. A java web-application is deployed on Google cloud and examined of its performance, further in this paper.
\end{abstract}

\section{General Terms}

Cloud Computing, Google-App-Engine, Java WebDeployment.

\section{INTRODUCTION}

Cloud Computing is a service used over a network for multipurposes like software, platform, infrastructure service and provides a better a way for virtualization in the IT field.[1] There are several fields that are affected by the cloud computing like deployment. Cloud Computing makes the IT fields enable for better performance using lesser resources. It includes delivery of the application as services throughout the internet and the software that provide services in the datacentre and hardware and the prototype shift. The data centresoftware and hardware is known as a cloud. [2]

Many of the companies are shifting to the cloud services like Google App Engine has been started by Google. Microsoft started Windows Azure, Amazon started EC2. A WebApplication is deployed on Google App Engine as a sample application. There are several terms which are discussed as follows:

\section{WHAT A CLOUD IS:}

A cloud is a pool of virtualized computer resources. [3]

A cloud can multitude a range of different loads, including wedge-style back-end works and collaborating, User-facing applications. It allows loads to be located and scaled-out rapidly through the quick provisioning of Virtual machines or somatic machines. It supports completed, self-recovering, extremely accessible programming prototypes those allow loads to improve from many obvious hardware/software disasters. It observers resource use in real time to enable rebalancing of provisions when desired.

A Cloud is an implicit world available for applicationsdeployment with optimized cost, whereas Cloud Computing is a regular word for anything that involves distributing services over the Internet. At its humblest, it is providing the assets and proficiencies of information technology enthusiastically as a service. Cloud Computing is a style of computing in which enthusiastically accessible and often virtualized assets are delivered as a service over the Internet. [4]

ADVANTAGES OF CLOUD COMPUTING:

- It is swift, with ease and speed of deployment.

- Its cost is use-based, and will likely be abridged.

- In house IT costs are condensed.

- Capital investment is cheap.

- The latest technology is offered always.

- The use of standard technology is optimistic and facilitate.

\section{CLOUD SERVICES:}

A cloud is a pool of systems, resources and their classes that provides all facilities as per the user-end's requirements. All the resources, applications are part of a cloud. [5] Cloud Computing provides following classification of its services:

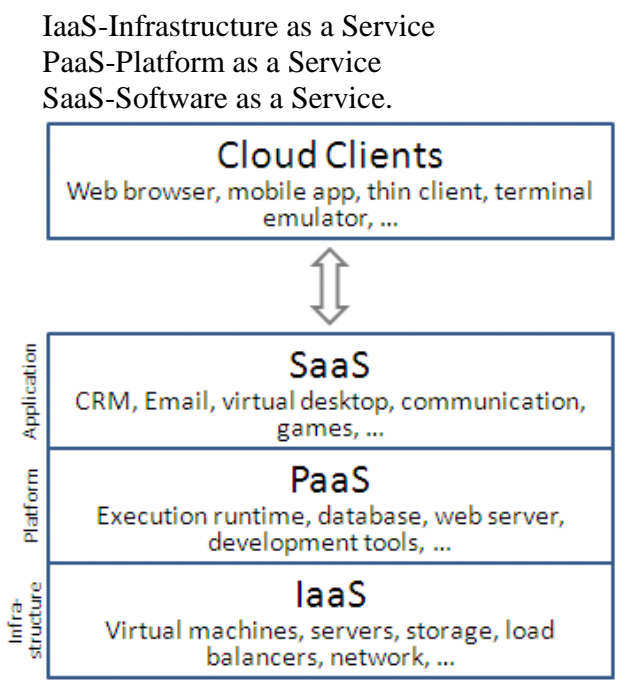

1.) Infrastructure as a Service

The IaaS is further classified into:

i.) Computation as a Service (CaaS):

In this kind of service, the virtual machine servers are lent. The cost of the virtual machine servers are based on the capacity of the machine like memory attributes of the server, its operating system and all deployment features. [6]

ii.) Data as a Service (DaaS):

In this kind of service, Storage is provided for all end-users for storing data. The cost estimation of the service is based on the scale of Gigabyte (GB) and decided by the provider. [7] 
There are several cloud computing platforms for the world. The Cloud Computing platform for Google is Google App Engine, which has an efficient and better system for deployment and all. [8]

Google has provided a standard all-inclusive answer to Cloud Computing, known as the Google App Engine. Google App Engine provides several features for its clients such as fine grained computing, data storage, data transfer etc. Google App Engine provides VPN (Virtual Private Network), elastic IP-Addressing etc. Google App Engine has become a standard model in Cloud Computing. [9]

2.) Platform as a Service

Platform as a Service (PaaS) provides an environment which is good in performance. Platform like OS could also be used over a network (internet) by any end-users at its requirement. [10] There would be no need of having an installed OS; one could load its OS using the PaaS. The Applications could also be served as a platform services.

Microsoft has provided Windows Azure as cloud computing servers. Windows Azure is an effort to provide PaaS services to the users. Window Azure Platform (WAP) is the cloud-OS offered by Microsoft. WAP includes several services with the cloud-OS. Azure services as virtual machine servers as its runtime environments.

\section{3.) Software as a Service}

As PaaS, SaaS provides Software as a Service on a cloud platform. Using SaaS, software may have been developed, installed and updated on the end-user's request. It reduces the management costs and the software conventions with a Rent Model. [11]

\section{RELATED WORK}

As per the growing development of Cloud Computing, it is going to be the future of the IT-Field. But there are several sections in which the cloud computing is still facing problems. Some of the issues are Performance, Security, Cost, Reliability etc. If the cloud computing wants to be the acceptable future in the IT field, it would have to overcome these issues. In this paper, the performance issue is examined and tried to resolve. To examine the performance issue, a java web-application is deployed to a cloud server (Google App Engine) [12] as follows:

\section{STEPS TO DEPLOY A JAVA WEB-APPLCATION ON GOOGLE APP ENGINE:}

STEP 1: A Java Web-Application can be deployed on Google App Engine using the appengine-java-sdk tool. First download the appengine-java-sdk tool.

STEP 2: Using the appcfg.cmd file, the application can be deployed. Assume the application name is MySampleApplication, the command would be:

appcfg.cmd update //*PATH TO APPLICATION FOLDER *// MySampleApplication

STEP 3: The Sample Web-Application is deployed now and would be available on app-name.appspot.com for ease access. The Application Administration can be accessed on appengine.google.com. [13]

Thus a java web-application is deployed on cloud server (Google App Engine). The performance issue is discussed in this paper which is a major issue in cloud computing for its users. The performance issue includes the delay for running the application on cloud server. The web-server sends its output of the web-application to the client. If the cloud server takes longer time to run the application, this would lead to a delay and this issue is discussed in this paper.

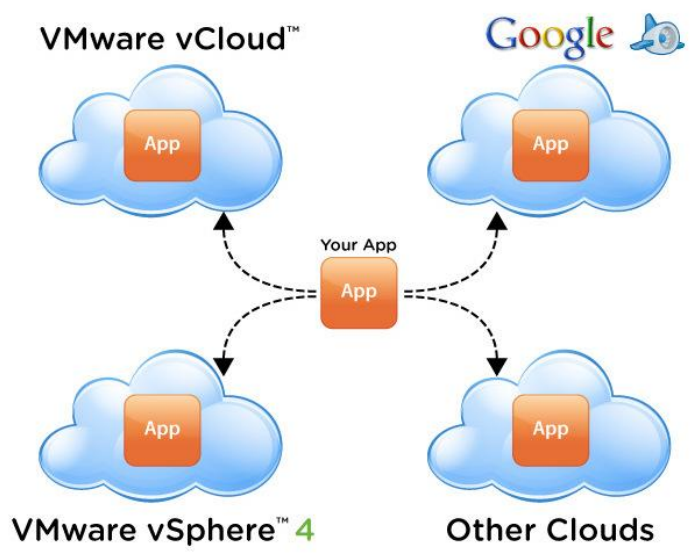

\section{ISSUES WITH WEB-DEPLOYMENT ON CLOUD}

There are several issues in Web-Deployment in cloud computing. As per the current research, the cloud computing is still facing challenges in its fields. Some of the issues are specified here, one of those would be tried to overcome:

\subsection{Performance Issue:}

This kind of issues is for those users which are far away from cloud in the factor of distance. The cloud may affect with its lesser performance. The root cause of this issue can be due to High-Latency-Delay. [14]

\subsection{Security Issue:}

This kind of issue has been a primary issue in IT. Cloud Computing is also facing this issue. Security attacks and threats are still the problem for the Servers. Several Kinds of security ideas may be used to avoid these issues. [15]

\subsection{Cost Issue:}

The cost estimation is measured on the basis of Bandwidth. If the Application needs large bandwidth, the cost would be increased for these kinds of applications. Small Applications need lesser bandwidth, their costs aren't an issue. Large Applications faces these kinds of issues. [16]

\subsection{Reliability Issue}

This issue includes the reliability of the cloud. The cloud is reliable if and only if its infrastructure and its resources are reliable. These kinds of issues are resolved through individual resources of the clouds. [17]

These issues are basic problems in the cloud computing. The area of the problem resolving would be the Performance Issue in the Cloud Computing. This kind of issue could be resolved by the filtering of individual applications. The WebApplication used in the Cloud would be a Java-WebApplication. The Java Cloud requirements are continuously growing better and more refined.

All Popular clouds are shifting due to the experimental results. In Cloud Computing, the loads ad costs are not definite in the market, so the prices seem to be changing, every so often in remarkable ways. Even the cloud sellers hasn't fixed the cost, they are only guessing the costs, like any X Dollars for $\mathrm{Y}$ Transactions. [18] 
Like in Google App Engine, the biggest problem for developers will be adjusting to Google's non-relational data

\section{OBJECTIVE}

As per the given Problem Formulations, the basic objective will be to determine the less-performance causes in Java WebApplication on Clouds.

- Following phases would be useful in Performance issues:

- Choosing a suitable platform for java application like Google App Engine etc.

- The Cloud Framework (i.e. The App Engine web-app Framework for Google App Engine) should be configured as per the requirement of the J2EE application. [19]

- The infrastructure of the cloud services should be more specific to the Java-Applications.

Following methods can be used for the above proposed objective:

* The Structure of the Cloud Computing in the respect of Deployment should be in a good manner. The Framework used in the Virtual Machine Server should be independent and optimized so that could be good at performance.[20]

* The resources which is used in the cloud computing should be single independent resource and should mention the required configuration (hardware and software) [21] and should be platform independent a reliable to use at large scale.

These issues would be resolved by several possible solutions. In the cloud services, to perform some specific task, there are several resources arranged in a particular order. [22] The Cloud Computing provides us customizing its resources according to the individual application and its use.

\section{METHODOLOGY}

The Methodology will used to overcome this issue would be:

- Optimization in Framework:

As per the Google app engine, the cloud computing has its dependency on the framework of the Web-Application. So the very first approach would be followed the optimization of the framework of the application in a cloud. The only framework, which has been customized for the cloud computing is Spring Framework yet now. [23]

While using Spring Framework in cloud deployment, if the application takes a longer time to load, would be thrown as DeadlineExceededException and the control will be shifted to the framework and now framework would take the respective decisions. [24] For a better Cloud Deployment, the entire framework should be optimized.

- Reducing or Avoiding the use of module Scanning: In Google App Engine, The Spring Framework process a set of observation as a signal-flag to any other object in its execution .Sometimes the requested resource cannot be availed due to the resource sharing, this also restricts the application-speed in its performance. The Component Scanning is also responsible for making the application with lesser performance and lesser efficient due to its time taking process. To avoid this problem, the Component Scanning would be avoided. In the mandatory case of using Component Scanning, it would be reduced. [25] stores. When Google App Engine was introduced, there were not so many database-projects in the market for clouds. This Methodology would work with JAVA WebApplications that are implemented in Spring Framework.

\section{CONCLUSION}

According to the results, the performance issue can be easily handled by filtering the web-application individually and the framework-customization. This issue has been a major issue for the cloud-users which would be handled by the suggested idea. This would decrease the High Latency Delay for the application performance and the application would not take longer time to run on the cloud server.

\section{ACKNOWLEDGMENTS}

Our thanks to the parents who motivated us and Mr. Sandeep Bhargav (Department of Computer Science), Gyan Vihar School of Engg. And Tech. who have contributed towards development of this issue

\section{REFERENCES}

[1] The Basics of Cloud Computing by Alexa Huth and James Cebula.

[2] Introduction to the cloud computing by Dialogic.com

[3] G. Juve, E. Deelman, K. Vahi, and G. Mehta, "Scientific Workflow Applications on Amazon EC2," Workshop on Cloud-based Services and Applications in conjunction with 5th IEEE International Conference on e-Science (eScience 2009), 2009.

[4] Using Google App Engine-O'Reilly Google Press by Charles Severance.

[5] X-as-a-Service: Cloud Computing with Google App Engine, Amazon Web Services, Microsoft Azure and Force.com by Rabi Prasad Padhy, Manas Ranjan Patra and Suresh-Chandra-Satapathy.

[6] Identifying Key Challenges in Performance Issues in Cloud Computing by Ashraf Zia.

[7] Cloud Computing Security Issues by Randy Marchany.

[8] The Cost of a Cloud: Research Problems in Data Center Networks by Albert Greenberg, James Hamilton, David A. Maltz, Parveen Patel.

[9] Cloud Computing: Security and Reliability Issues by Farhad Ahamed, Seyed Shahrestani and Athula Ginige.

[10] A Novel Approach for Handling Security in Cloud Computing Services by Sahar Mohammad Abduljalil, Osman hegazy and Ehab E Hassanein.

[11] SAP Cloud Computing by Joseph Yeruva, MPHASIS

[12] Cloud Computing in the Public Sector by Russell Craig, Jeff Frazier, Norm Jacknis, Seanan Murphy, Carolyn Purcell, Patrick Spencer, JD Stanley.

[13] Moving from Legacy Systems to Cloud Computing: A Tata Communication White Paper.

[14] GTSI Group, "Cloud Computing-Building a Framework for Successful Transition," White Paper, GTSI Corporation, 2009.

[15] Rajnish Choubey, Rajshree Dubey and Joy Bhattacharjee, "A Survey on Cloud Computing Security, Challenges and Threats", International Journal on 
Computer Science and Engineering (IJCSE), vol. 3, No. 3, 2011 .

[16] Andrew Joint and Edwin Baker, "Knowing the past to understand the present- issues in the contracting for cloud based services", Computer Law and Security Review 27, pp 407-415, 2011.

[17] Michael Miller, "Cloud Computing Pros and Cons for End Users", microsoftpartnercommunity.co.uk, 2009.

[18] Radu Prodan and Simon Ostermann, "A Survey and Taxonomy of Infrastructure as a Service and Web Hosting Cloud Providers", 10th IEEE/ACM International Conference on Grid Computing, 2009.

[19] Google App Engine documentation link. http://code.google.com/appengine/docs/whatisgoogleapp engine.html.

[20] Liladhar R. Rewatkar, Ujwal A. Lanjewar, Implementation of Cloud Computing on Web Application, International Journal of Computer Applications (0975 - 8887) Volume 2, No.8, June 2010.

[21] K. Mukherjee, G.Sahoo, Development of Mathematical Model for Market-Oriented Cloud Computing,
International Journal of Computer Applications (0975 8887) Volume 9- No.11, November 2010.

[22] Google App Engine And Performance Of The Web Application By Anjali Jain.

[23] Into the Cloud: An Evaluation of the Google App Engine by Chornyi, Dmitry, Riediger, Julain, Wolfenstetter, Thomas.

[24] Mei, L., Chan, W. K., and Tse, T. H. 2008 A tale of clouds: paradigm comparisons and some thoughts on research issues. In Proceedings of the 2008 IEEE AsiaPacific Services Computing Conference, pp. 464-469.

[25] Raman, T. V. 2008. Cloud computing and equal access for all. In Proceedings of the International CrossDisplinary Workshop on Web Accessibility, 2008, pp. 14.

\section{AUTHOR'S PROFILE}

Ankit Kumar Sahu, born on $26^{\text {th }}$ August, 1991 in Jaipur, Rajasthan, India. He completed his bachelor's degree (B. Tech.) in Computer Science from Suresh Gyan Vihar University, Jaipur in 2012. He is currently pursuing his master's (M. Tech.) in Software Engineering from Suresh Gyan Vihar University, Jaipur. 